\title{
A meta-analysis of combination therapy versus single-agent therapy in anthracycline- and taxane- pretreated metastatic breast cancer: results from nine randomized Phase III trials
}

\author{
This article was published in the following Dove Press journal: \\ OncoTargets and Therapy \\ 4 July 2016 \\ Number of times this article has been viewed
}

\author{
Liang $X u^{1,2, *}$ \\ Xiaobo $\mathrm{Wu}^{3, *}$ \\ Chun $\mathrm{Hu}^{1,2}$ \\ Zhiying Zhang ${ }^{4}$ \\ Le Zhang ${ }^{1,2}$ \\ Shujing Liang ${ }^{1,2}$ \\ Yingchun $\mathrm{Xu}^{5}$ \\ Fengchun Zhang ${ }^{1,2}$ \\ 'Department of Oncology, Suzhou \\ Kowloon Hospital, Shanghai Jiaotong \\ University School of Medicine, Suzhou, \\ ${ }^{2}$ Department of Oncology, Ruijin \\ Hospital, Shanghai Jiaotong University \\ School of Medicine, Shanghai, \\ ${ }^{3}$ Prevention and Cure Center of \\ Breast Disease, Third Hospital of \\ Nanchang, Nanchang, ${ }^{4}$ Graduate \\ School, Xuzhou Medical College, \\ Xuzhou, ${ }^{5}$ Department of Oncology, \\ Renji Hospital, Shanghai Jiaotong \\ University School of Medicine, \\ Shanghai, People's Republic of China \\ *These authors contributed equally \\ to this work
}

Correspondence: Yingchun Xu

Department of Oncology, Renji Hospital,

Shanghai jiaotong University School of

Medicine, 1630 Dongfang Road, Shanghai

200 I27, People's Republic of China

Tel +86 I358557 6486

Email84985540@qq.com

Fengchun Zhang

Department of Oncology, Suzhou

Kowloon Hospital, Shanghai Jiaotong

University School of Medicine,

I 8 Wansheng Street, Suzhou

21502I, People's Republic of China

Tel +86 I86 2II795347

Email fczhang2005@163.com
Abstract: Nowadays, the philosophy of treating metastatic breast cancer (MBC) is slowly evolving. Especially for the anthracycline- and taxane-pretreated MBC patients, no standard therapy exists in this setting. Whether to choose doublet agents or single agent as salvage treatment remains fiercely debated. Thus, we conducted a meta-analysis to resolve this problem. Databases including PubMed, EMBASE, and Cochrane library were searched for Phase III randomized clinical trials (published before August 2015) comparing the efficacy and adverse effects between the combination therapy and single-agent therapy in anthracycline- and taxanepretreated $\mathrm{MBC}$ patients. The primary end point was the overall survival (OS), and the secondary end points were the progression-free survival (PFS), overall response rate (ORR), and grade 3 or 4 toxicities. The pooled hazard ratio (HR) and pooled risk ratio (RR) were used to evaluate the efficacy. Analyses were also performed to estimate the side effects and safety of both groups. In all, nine eligible randomized clinical trials were included in this meta-analysis. Improvements were proven in the doublet agents group on OS (HR $0.90,95 \%$ confidence interval [CI] 0.84-0.96, $P=0.002$ ), PFS (HR 0.81, 95\% CI 0.76-0.88, $P<0.001$ ), and ORR (RR 1.72, 95\% CI 1.34-2.21, $P<0.001)$. Notably, subgroup analysis failed to favor the targeted agentbased combination in terms of OS (HR 1.08, 95\% CI 0.89-1.31, $P=0.365$ ), PFS (HR 1.09, 95\% CI 0.88-1.35, $P=0.433$ ), and ORR (RR 1.60, 95\% CI 0.69-3.71, $P=0.278$ ) compared with single agent. In addition, although more hematological and gastrointestinal toxicities were observed in the doublet agents group, they were acceptable and manageable. Taken together, when compared with single-agent therapy, doublet agents should be considered a treatment option because of the superior efficacy and the manageable safety profile for the prior anthracycline- and taxane-treated MBC patients.

Keywords: breast cancer, anthracyclines, chemotherapy, combination therapy, toxicity

\section{Introduction}

Breast cancer is one the most common life-threatening malignancies worldwide. ${ }^{1}$ Despite great efforts having been made to explore the therapeutic effect for breast cancer, a substantial proportion of patients experience disease progression and metastasis, which indicates an adverse prognosis. For the metastatic breast cancer (MBC) patients, chemotherapy regimens containing taxanes and anthracyclines are the first-line standard of care. ${ }^{2}$ However, anthracyclines and taxanes are routinely used as standard adjuvant or neoadjuvant treatment for high-risk early breast cancer, thereby limiting their use in patients who subsequently develop metastasis. ${ }^{3}$ Therefore, these agents are 
generally not considered for these MBC patients due to the potential problems associated with cumulative cardiotoxicity and drug resistance. ${ }^{4}$ Consequently, there is no standard treatment for MBC patients whose disease progressed prior to anthracycline- and taxane-based chemotherapy to date, and it remains a great challenge to select an appropriate regimen in this setting.

Recently, various agents including antitubulins (eg, vinorelbine, ${ }^{5,6}$ ixabepilone, ${ }^{7}$ and eribulin ${ }^{8}$ ), antimetabolites (eg, capecitabine $\mathrm{e}^{9,10}$ and gemcitabine ${ }^{11,12}$ ), topoisomerase I inhibitors (eg, irinotecan ${ }^{13}$ ), and platinum analogs (eg, cisplatin $^{14}$ ) have been evaluated for their efficacy and safety in this setting when used either alone or in combination with other cytotoxic agents. Compared with single agent, some studies found that doublet agents could gain additional clinical benefits including progression-free survival (PFS), overall survival (OS), and overall response rate (ORR), ${ }^{7,11}$ which could offer an option for women with anthracyclineand taxane-pretreated MBC. Nevertheless, by contrast, some studies revealed that combination therapy contributed to more serious toxicities such as neutropenia, leukopenia, anemia, and constipation without any noticeable improvements in clinical benefit. ${ }^{12,15}$ Hence, optimal treatments for advanced breast cancer or MBC previously treated by anthracyclines and taxanes remain controversial. Most physicians usually select a regimen based on their own experience and clinical judgment.

In an attempt to resolve this problem, we performed a meta-analysis of Phase III randomized clinical trials (RCTs) comparing the efficacy and toxicity of combination therapy with single-agent therapy in those MBC patients who had been heavily pretreated with anthracyclines and taxanes. We also aimed to assess the comparative efficacy and side effects of targeted drug-based combination therapy and single-agent therapy in this setting.

\section{Methods}

\section{Literature search strategy and inclusion criteria}

RCTs that were published in English and had compared doublet agents with single agent in anthracycline- and taxane-pretreated MBC were identified between January 1980 and August 2015 from the following databases: PubMed, EMBASE, and Cochrane library. For ongoing studies, we searched the ClinicalTrails.gov network (https:// ClinicalTrials.gov). The reference lists of the original articles were also searched for relevant studies. In addition, posters from the American Society of Clinical Oncology (ASCO) annual meetings and the European Society of Medical Oncology (ESMO) in the past 10 years and posters from the San Antonio Breast Cancer Symposium (SABCS) between 2009 and 2014 were also manually searched. The search algorithm used in the electronic database was (breast OR mammary) AND (carcinoma OR neoplasm OR tumor OR cancer) AND (metastatic OR relapse*) AND (pretreat*) AND (Phase III clinical trial). All titles, abstracts, and related citations were scanned and reviewed. This meta-analysis was conducted according to the Preferred Reporting Items for Systematic Reviews and Meta-analyses (PRISMA) statement. ${ }^{16}$

The inclusion criteria were: 1) Phase III RCTs; 2) eligible patients included in the studies were $>18$ years old with pathologically confirmed advanced breast cancer or MBC and pretreated with an anthracycline and/or a taxane as adjuvant or palliative treatment; 3) studies must compare the efficacy and toxicity of combination therapy with singleagent therapy; 4) the studies must have sufficient data for extraction, stratification, and calculation.

\section{Quality assessment of the studies}

Eligibility and quality of the studies were evaluated independently by two investigators. If any discrepancies existed, a third expert was involved, and a consensus was reached after discussion. In the case of more than one reported publication of the same trial, a decision was made to include only the most recent or the most complete report. Subsequently, an open assessment of trials was performed using the methods that were previously reported by Jadad et $\mathrm{al},{ }^{17}$ which assessed trials according to the following three queries: 1) Whether the trial reported an appropriate randomization method (0-2 scores); 2) whether the report included an appropriate blinding method (0-2 scores); 3 ) whether the report included an account of the number of withdrawals or dropouts.

\section{Data extraction}

Two investigators independently extracted the data and recorded these data on a standardized form, and any disagreement was resolved through discussion. We extracted the following information from each publication: 1) Basic information of each study such as journal name, authors' names, and publication year; 2) characteristics of the patients such as sex, age, performance status, disease burden, and ethnicity; 3) information of the study design 
such as samples per group, methods of randomization and blinded or open-label design, inclusion and exclusion criteria, types of end point analysis, and follow-up; 4) data such as the regimen and drug dose, withdrawal from therapy, PFS, OS, ORR, and incidence of adverse effects (AEs) were also extracted to evaluate the efficacy and toxicity of the regimens.

\section{Statistical analysis}

This data analysis was based on intention-to-treat population for efficacy and AEs. Pooled risk ratio (RR) and 95\% confidence interval (CI) were used to appraise the ORR and AEs. In addition, OS and PFS were evaluated by pooled hazard ratio (HR) analysis and the respective 95\% CI. RRs, HRs, and their respective 95\% CIs were obtained from the original article. If the HRs and 95\% CIs were not reported directly, we calculated them with the published methods. ${ }^{18,19}$ Engauge digitizer Version 4.8 was used to read and analyze the Kaplan-Meier curves of the included studies. This was a free software that can be downloaded from http://sourceforge.net/projects/digitizer/. HR calculation spreadsheet used to calculate HRs and 95\% CIs were freely downloaded from http://www.biomedcentral.com/content/ supplementary/1745-6215-8-16-S1.xls. Heterogeneity was estimated by $\chi^{2}$-based $Q$-test. ${ }^{20}$ When $P_{\text {heterogeneity }}<0.100$ or $I^{2}>50 \%$, heterogeneity was considered statistically significant. If heterogeneity existed, a random effect model was used. If not, a fixed effect model was used..$^{21,22}$ To better explore the source of heterogeneity, subgroup stratification analyses were performed based on several study characteristics, such as publication year, ethnicity, and different drug combinations. Egger's test and Begg's test were applied to evaluate the publication bias..$^{23,24}$ Moreover, sensitivity analysis was conducted by omitting one study at a time to evaluate the influence of a single study on the overall estimate. All the statistical calculations were completed by using STATA software (Version 10.1; StataCorp LP, College Station, TX, USA). A statistical test with a $P<0.05$ was considered significant. By convention, $\mathrm{HR}>1$ reflects more progression and death in the doublet agents compared with single-agent group; $\mathrm{RR}>1$ reflects more relevant adverse events and more treatment response in the doublet agents group, and vice versa.

\section{Results \\ Study search and eligibility}

The literature search process is shown in Figure 1. After electronic database research, 204 records that compared efficacy of doublet agents therapy versus single-agent therapy in the anthracycline- and taxane-pretreated MBC population were identified. In addition, three records were identified by searching the international breast cancer meeting reports, the reference list of original articles, and reviews. Of them, 63 duplicate publications were removed subsequently. In addition, 128 records were excluded by screening the titles, abstracts, and full texts based on our inclusion criteria (irrelevant: 75 records, review: 33 records, meta-analysis: four records, and others: 16 records). Finally, 16 records were

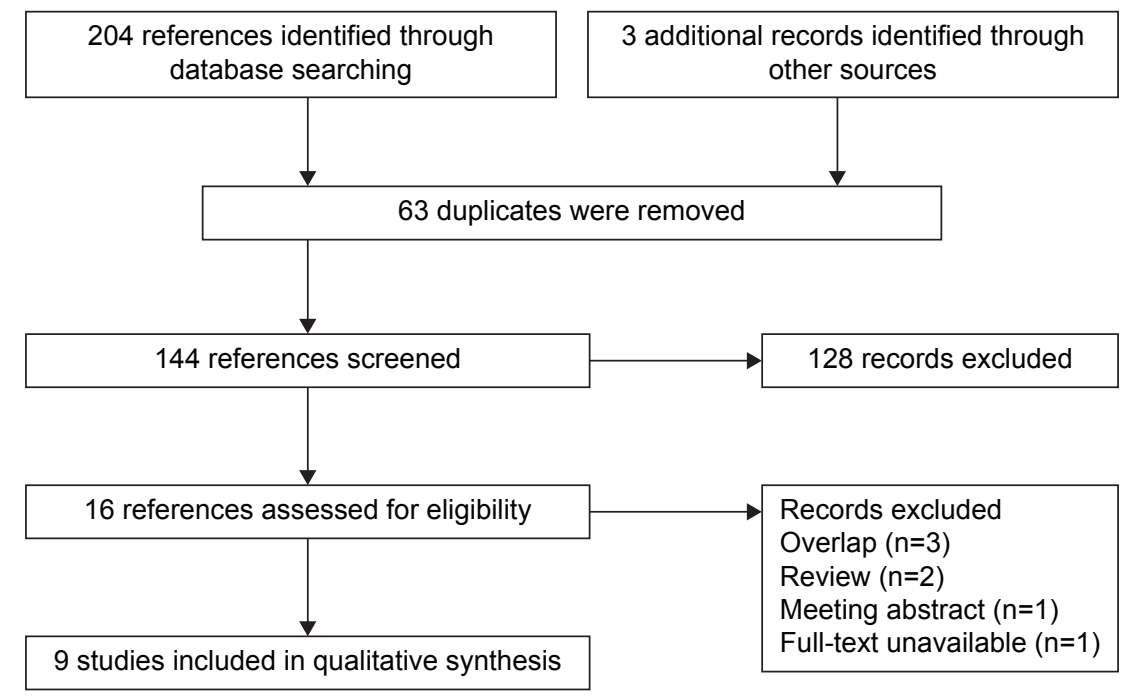

Figure I Flow diagram and results of literature review.

Note: Flow diagram depicts the screening process, including the number and reason of inclusion and exclusion. 
assessed for eligibility. Seven of them were then excluded: three overlapping studies, two peer-reviewed articles, one meeting abstract, and one full-text article that was unavailable. Therefore, nine studies were ultimately included in this meta-analysis. ${ }^{7,11,12,15,25-29}$

\section{Characteristics of the trials}

All nine trials included in this meta-analysis were Phase III RCTs that were published from 2002 to 2013, and 4,641 MBC patients pretreated with anthracyclines and taxanes were involved in the further meta-analysis. Of them, 2,315 were randomly assigned to doublet agents therapy and 2,326 patients were randomly assigned to single-agent therapy. Of the nine trials included, seven trials compared the efficacy and safety of combination chemotherapy with single-agent therapy. ${ }^{7,11,12,25,27,28}$ However, the remaining two evaluated the efficacy of chemotherapy plus targeted therapy versus single-agent therapy. ${ }^{15,26}$ The baseline characteristics of the eight trials are shown in Table 1.

\section{Overall survival}

We estimated the HRs and 95\% CIs for all nine studies for overall survival. Of them, six studies reported HRs and their $95 \%$ CIs for OS directly in the original paper., ${ }^{71,15,27,28}$ In the remaining three studies, HRs and 95\% CIs were calculated using the original Kaplan-Meier curve data. ${ }^{12,25,26}$ No obvious heterogeneity existed ( $P_{\text {heterogeneity }}=0.207, I^{2}=26.7 \%$ ); and therefore, a fixed effects model was used to calculate the pooled HR. After qualitative synthesis, doublet agents appeared to have a more favorable overall survival compared with single agent (HR 0.90, 95\% CI 0.84-0.96, $P=0.002$, Figure 2A). However, in our further subgroup analysis depending on different drug combinations, we found that targeted drug plus chemotherapy did not improve OS compared with single drug therapy ( $\mathrm{HR}=1.08,95 \% \mathrm{CI}$ $0.89-1.31, P=0.423$, Figure 2A). Next, we also demonstrated the ixabepilone-based combinations result in superior overall survival compared with single agent alone (HR 0.87, 95\% CI $0.79-0.96, P=0.008$, Figure S1A).

\section{Progression-free survival}

In the nine studies that we assessed, the PFS data of two were unavailable. ${ }^{25,27}$ Additionally, in the remaining seven studies, HRs and $95 \%$ CIs of two were calculated from the curve data because the PFS data were not directly reported. ${ }^{11,12}$ In our further meta-analysis, an enhanced PFS survival was also observed in the combination therapy group (PFS: HR 0.81, 95\% CI 0.76-0.88, $P<0.001)$. The random effect model was used because of the obvious heterogeneity in the overall PFS

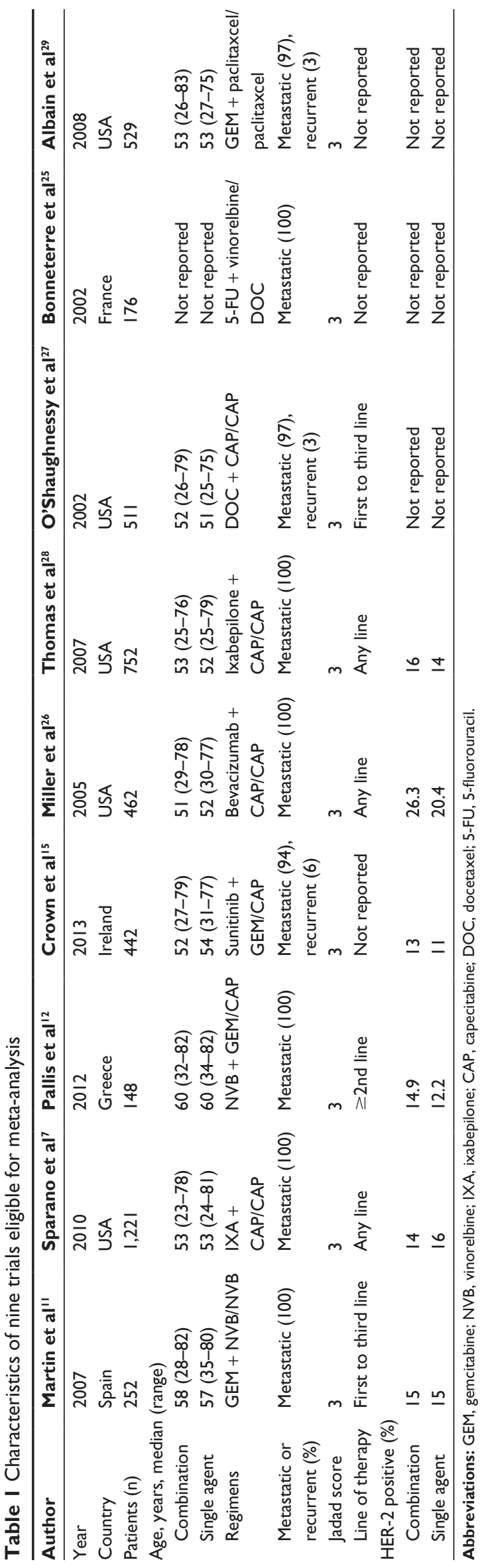


A

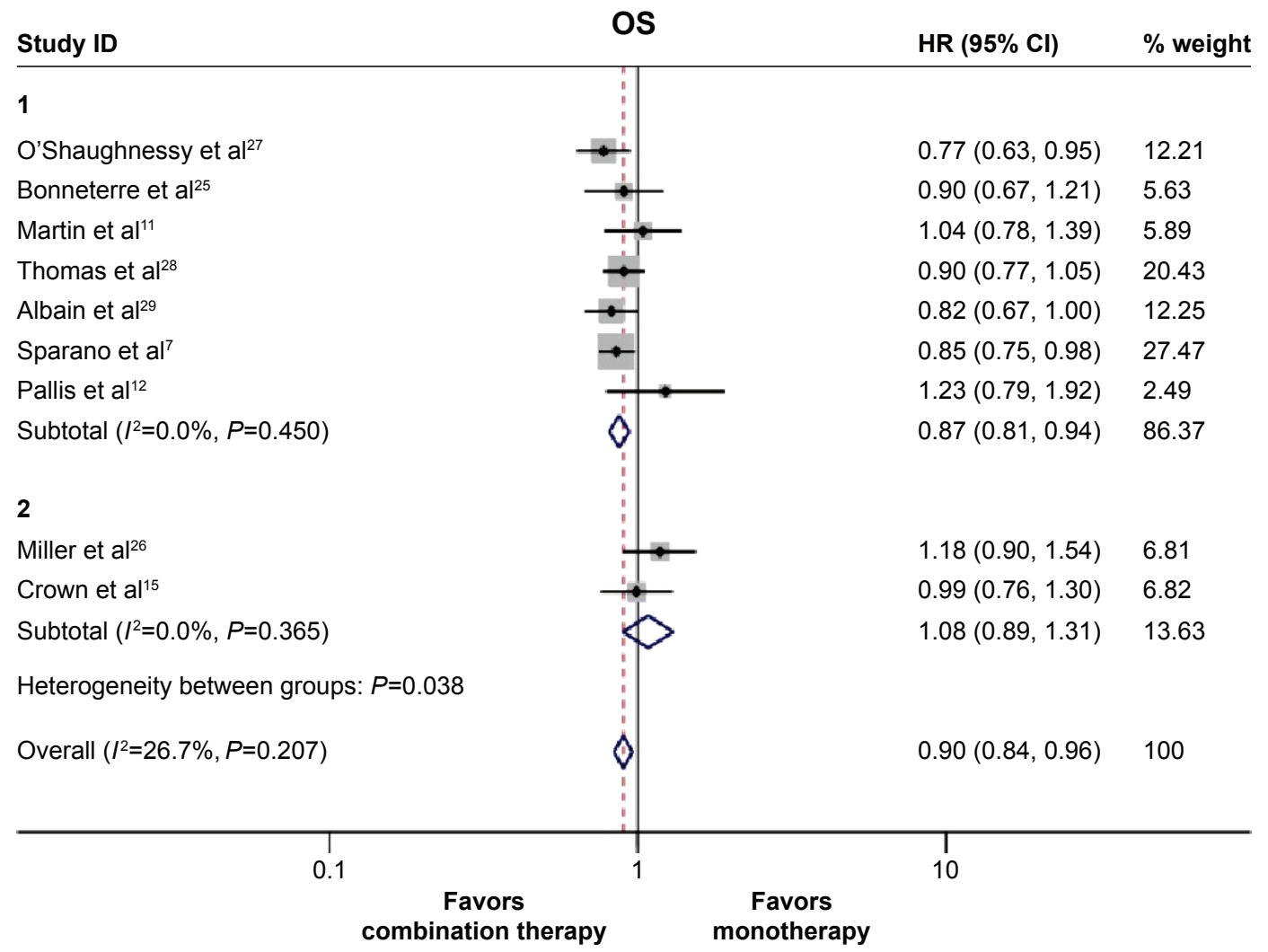

B

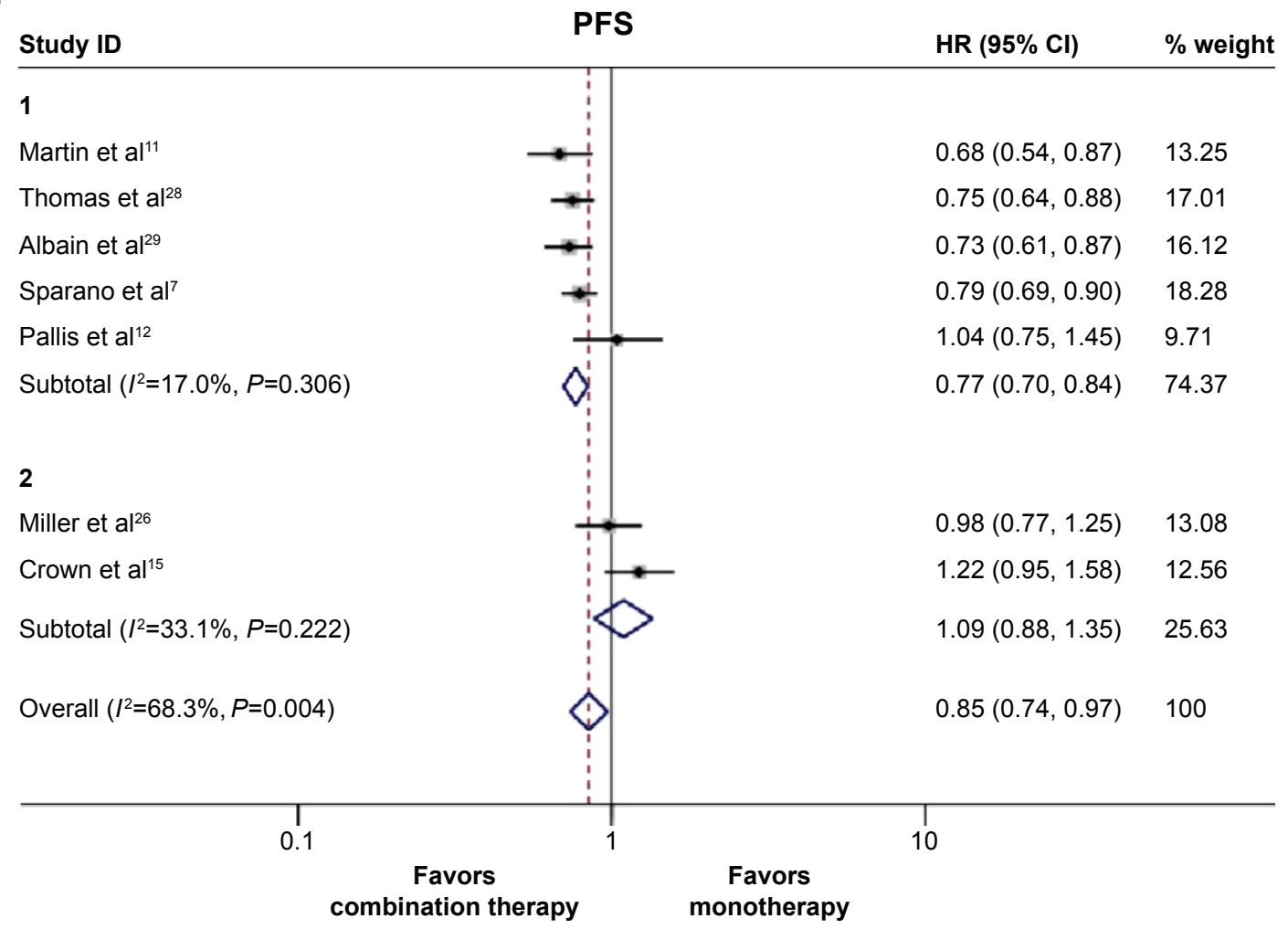

Figure 2 The pooled hazard ratio $(\mathrm{HR})$ and $95 \% \mathrm{Cl}$ for survival following doublet agents therapy and single-agent therapy.

Notes: The size of the square box is proportional to the weight that each study contributes in the meta-analysis. The overall estimate and confidence interval are marked by a diamond. Symbols on the right of the solid line indicate HR $>$ I, and symbols on the left of the solid line indicate HR $<$ I. (A) Overall survival. (B) Progression free survival. For the subgroups, group I represents the studies that compared the two chemotherapy agents combination with single-agent therapy, and group 2 represents the studies that compared the targeted therapy plus chemotherapy with single-agent therapy. The HR and $95 \% \mathrm{Cl}$ for OS and PFS were calculated with a fixed effect model. Weights are from fixed effect model. Abbreviations: $\mathrm{Cl}$, confidence interval; OS, overall survival; PFS, progression-free survival. 
$\left(I^{2}=68.3 \%, P=0.004\right.$, Figure $\left.2 \mathrm{~B}\right)$. Further subgroup analysis was also performed, we observed different drug combinations partly contributed to the observed heterogeneity. In the two chemotherapy drugs combined subgroup, the pooled HR indicated a significant improvement in PFS compared with single-agent arm (HR 0.77, 95\% CI 0.70-0.84, $P=0.000$, Figure 2B), whereas PFS did not improve with targeted therapy-based combination therapy compared with single-agent therapy (HR 1.09, 95\% CI 0.88-1.35, $P=0.433$, Figure 2B). Furthermore, we also found ixabepilone-based combinations significantly improved superior PFS compared with single agent alone (HR 0.77, 95\% CI 0.70-0.86, $P<0.001$, Figure S1B).

\section{Overall response rate}

The ORR data were available in all nine trials. Owing to the significant heterogeneity that existed $\left(P_{\text {heterogeneity }}=0.004\right.$, $I^{2}=64.6 \%$ ), the pooled RR was calculated using the random effect model. We found that the doublet agents showed a significantly improved ORR when compared with single agent (35.0\% vs 23.0\%, RR 1.72, 95\% CI 1.34-2.21, $P<0.001$, Figure 3); Furthermore, we also performed subgroup analysis to evaluate the source of this heterogeneity. We identified that different drug combinations could partly explain the observed heterogeneity. In the anthracycline- and taxane-pretreated MBC population, the two chemotherapy drugs combination regimen (without any targeted drug in the regimen) appeared to have a superior response rate ( $R R$ $1.76,95 \%$ CI 1.34-2.31, $P<0.001$, Figure 3) when compared with single-agent therapy. Moreover, consistent with PFS and OS, we also found that chemotherapy plus targeted therapy did not elicit a superior response rate when compared with the single agent (RR 1.60, 95\% CI $=0.69-3.71, P=0.278$, Figure 3). However, ixabepilone-based combinations significantly improved response rate when compared with single agent (RR 2.42, 95\% CI 1.46-4.02, $P<0.001$, Figure S2). This suggests that different drug combinations also contribute to the heterogeneity of ORR.

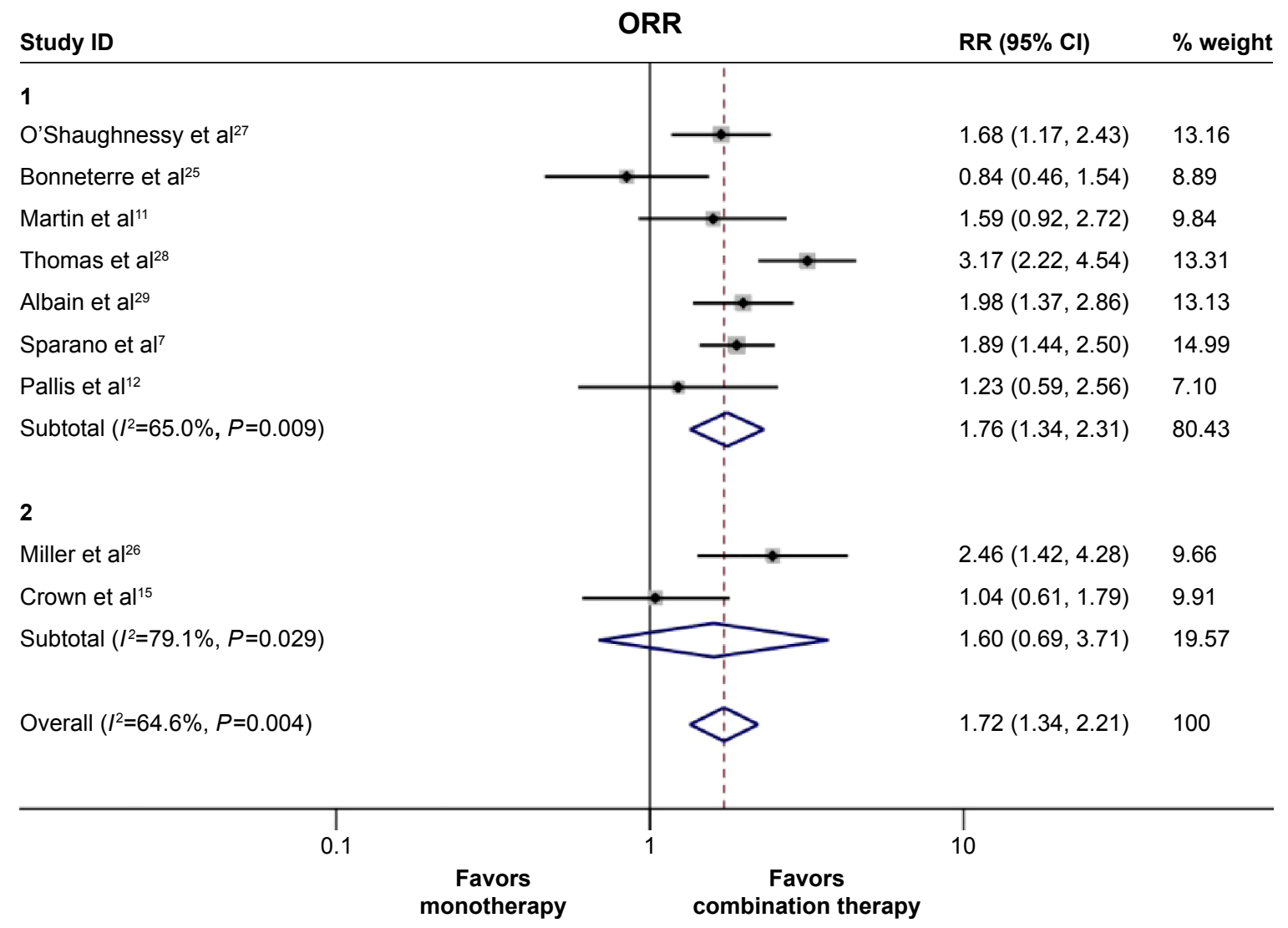

Figure 3 Pooled risk ratio (RR) and $95 \% \mathrm{Cl}$ for overall response rate.

Notes: The size of the square box is proportional to the weight that each study contributes in the meta-analysis. The overall estimate and confidence interval are marked by a diamond. Symbols on the right of the solid line indicate RR $>$ I, and symbols on the left of the solid line indicate RR $<$ I. The RR and $95 \% \mathrm{Cl}$ were calculated with a random effect model. For the subgroups, group I represents the studies that compared the two chemotherapy agents combination with single-agent therapy, and group 2 represents the studies that compared the targeted therapy plus chemotherapy with single-agent therapy. Weights are from random effect analysis.

Abbreviations: $\mathrm{Cl}$, confidence interval; ORR, overall response rate. 


\section{Safety}

Concerning the grade 3 or 4 hematological toxicities, leukopenia, anemia, neutropenia, thrombocytopenia, and febrile leukopenia were more frequent in the doublet agents group (Table 2). Similarly, doublet agents produced significantly increased gastrointestinal toxicities including nausea, stomatitis, and pharyngitis than did single agent, whereas the incidence of diarrhea and anorexia in the doublet agents did not differ from the single agent (Table 2). Additionally, for other toxicities, the difference in hand-foot syndrome and toxic death between the doublets agents group and single-agent group failed to reach statistical significance (Table 2). Taken together, these results suggested that the toxicities were manageable, although they were more frequent in the combination group. The pooled RRs of the most frequent toxicities between the two groups are shown in Figure 4A-F.

\section{Publication bias and sensitivity analysis}

To acquire acceptable reliability, we took some steps to evaluate any potential publication bias. First, our literature search strategy was extensive; second, the studies selected in this meta-analysis were strictly concordant with the inclusion and exclusion criteria; finally, the publication bias was evaluated by several methods. As shown in Figure $5 \mathrm{~A}-\mathrm{C}$, there was no obvious publication bias existed in this meta-analysis (ORR: Begg's test, $P=0.175$, Egger's test, $P=0.153$; PFS: Begg's test, $P=0.548$, Egger's test, $P=0.167$; OS: Begg's test, $P=0.175$, Egger's test, $P=0.051$ ).

In the present meta-analysis, sensitivity analyses were also performed by sequential removal of each eligible study to assess their influence on the pooled estimate. We found that the omission of several ixabepilone-containing studies ${ }^{7,28}$ clearly influenced the pooled estimates (Figure 6).
This observation indicated that the addition of ixabepilone to the regimen significantly influenced the pooled ORR/PFS/OS, which might lead to heterogeneity in the meta-analysis.

\section{Discussion}

For decades, despite significant advances in treating breast cancer patients, disease relapse and metastasis are still major problems..$^{30}$ Especially for those MBC patients who progressed after anthracycline- and taxane-based chemotherapy, there has been no standard care to date. Recently, many RCTs compared the efficacy and toxicity of various combinations of drugs with single-agent therapy in this setting, and the results were controversial. Furthermore, in 2013, a meta-analysis led by Qi et al ${ }^{31}$ compared the efficacies of the two treatment modalities for the anthracycline-pretreated MBC patients. Unfortunately, because only four RCTs were included in their study and the information was somewhat limited, they failed to clearly prove the role of combination therapy in the treatment of MBC pretreated with an anthracycline and/or a taxane. Therefore, we updated and summarized the information of related RCTs in order to aid decision making when cytotoxic agents were considered for these patients.

Consequently, nine RCTs were included in our metaanalysis ${ }^{7,11,12,15,25-28}$ instead of four in the previous metaanalysis (Figure 1), and the additional five studies enabled us to perform further subgroup analysis. Moreover, unlike the previous study that showed a trend in favor of doublet agents with respect to OS, but the difference was not statistically significant partly due to the limited RCTs included, we demonstrated for the first time that doublet agents were more efficacious in terms of OS (HR 0.90, 95\% CI 0.84-0.96, $P=0.002$ ), PFS (HR $0.81,95 \%$ CI $0.76-0.88, P<0.001$ ), and ORR (RR 1.72, 95\% CI 1.34-2.21, $P<0.001$ ) when

Table 2 Comparisons of grade 3-4 toxicities in the doublet agents group, with single-agent group, in this meta-analysis

\begin{tabular}{|c|c|c|c|c|c|c|c|}
\hline \multirow[t]{2}{*}{ Toxicity } & \multirow[t]{2}{*}{ Trials } & \multirow[t]{2}{*}{ Doublets } & \multirow{2}{*}{$\begin{array}{l}\text { Single } \\
\text { agent }\end{array}$} & \multicolumn{2}{|c|}{ Heterogeneity } & \multirow[t]{2}{*}{$\mathbf{R R}(95 \% \mathrm{Cl})$} & \multirow[t]{2}{*}{$P$-value } \\
\hline & & & & $P$-value & $I^{2}(\%)$ & & \\
\hline Anorexia & 5 & $27 / I, 500$ & $16 / 1,487$ & 0.239 & 27.4 & $\mathrm{I} .64(0.84-3.2 \mathrm{I})$ & 0.146 \\
\hline Diarrhea & 8 & $|55 / 2,09|$ & $159 / 2,079$ & 0.006 & 64.5 & $0.98(0.62-1.55)$ & 0.934 \\
\hline Nausea & 7 & $75 / 1,927$ & $32 / 1,919$ & 0.482 & 0.0 & $2.34(1.52-3.59)$ & 0.000 \\
\hline Stomatitis or pharyngitis & 8 & $1 \mid 5 / 2,140$ & $35 / 2,130$ & 0.114 & 39.7 & $3.32(2.22-4.95)$ & 0.000 \\
\hline Leukopenia & 4 & $597 / 1,399$ & $72 / 1,395$ & 0.000 & 89.5 & $7.82(2.45-24.97)$ & 0.001 \\
\hline Anemia & 8 & $115 / 1,956$ & $62 / 1,932$ & 0.608 & 0.0 & $1.85(1.34-2.56)$ & 0.000 \\
\hline Neutropenia & 8 & $I, 066 / I, 977$ & $288 / 1,973$ & 0.000 & 97.0 & $4.77(1.67-13.60)$ & 0.003 \\
\hline Thrombocytopenia & 5 & $90 / 1,401$ & $33 / 1,384$ & 0.675 & 0.0 & $2.75(1.82-4.14)$ & 0.000 \\
\hline Febrile leukopenia & 5 & $124 / 1,604$ & $70 / 1,638$ & 0.000 & 87.1 & $3.36(1.04-10.84)$ & 0.043 \\
\hline Toxic death & 5 & $15 / 1,325$ & $6 / 1,332$ & 0.821 & 0.0 & $2.4(0.89-6.44)$ & 0.051 \\
\hline Hand-foot syndrome & 6 & $351 / 1,735$ & $293 / 1,730$ & 0.000 & 84.2 & $1.29(0.74-2.22)$ & 0.366 \\
\hline
\end{tabular}

Notes: The RRs and 95\% Cls of anemia, anorexia, nausea, thrombocytopenia, and toxic death were calculated with fixed effect models. And the RRs and 95\% Cls of the other adverse effects were calculated with random effect models.

Abbreviations: $\mathrm{RR}$, risk ratio; $\mathrm{Cl}$, confidence interval. 

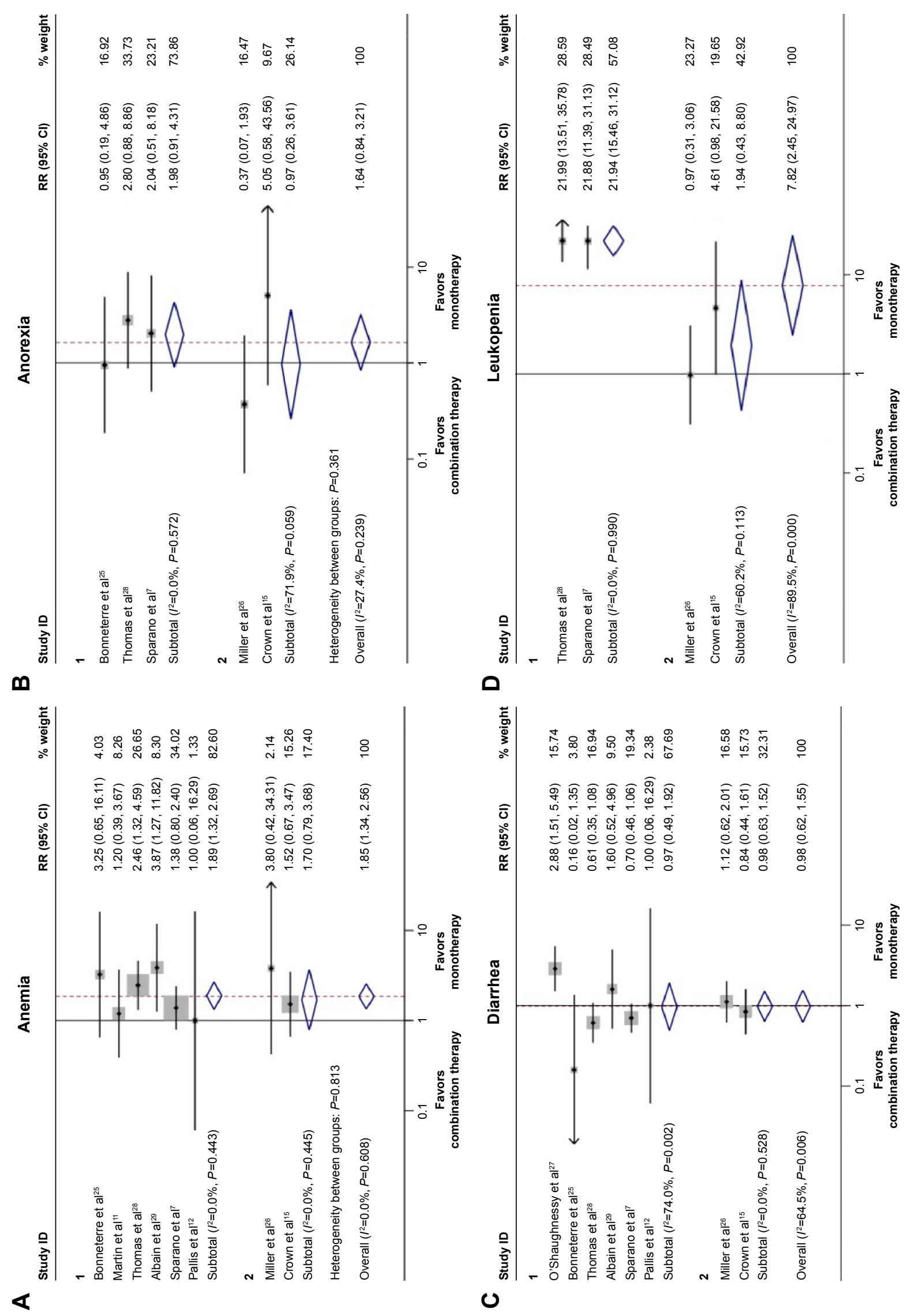


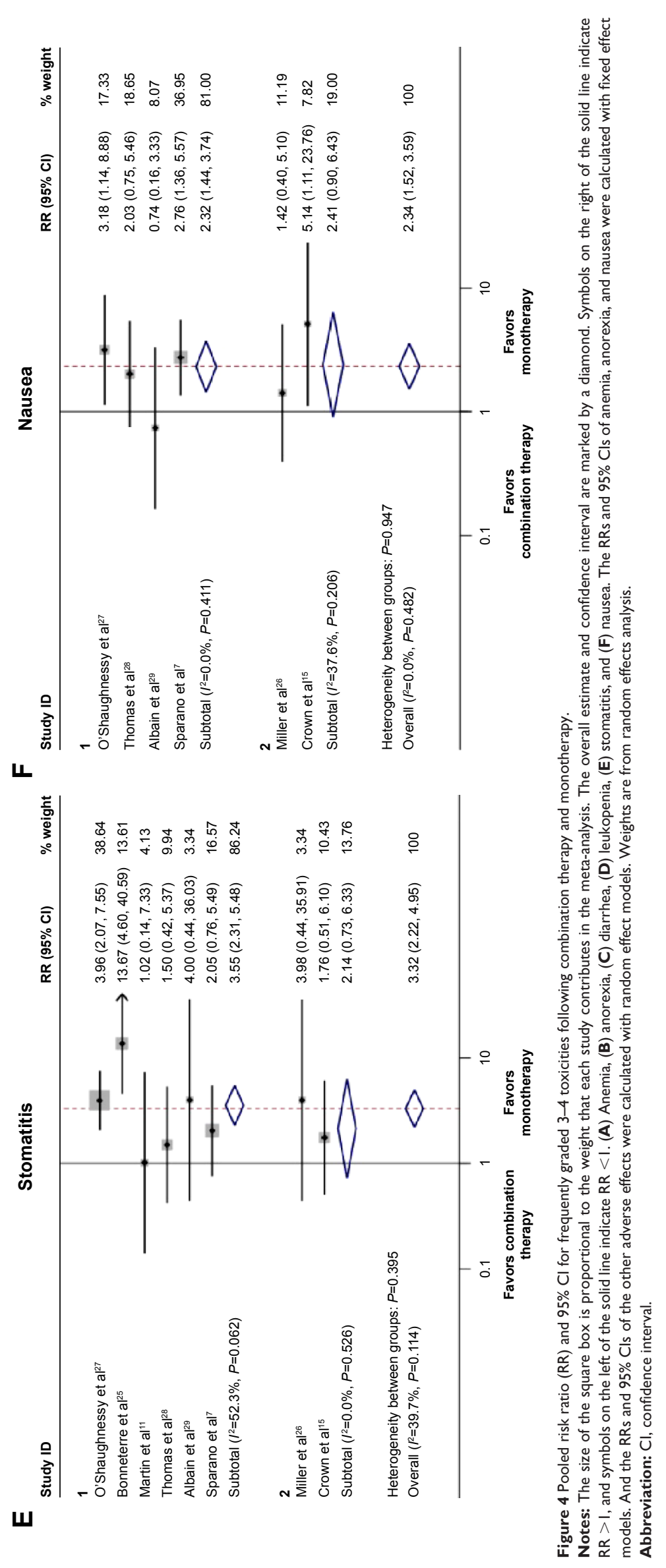



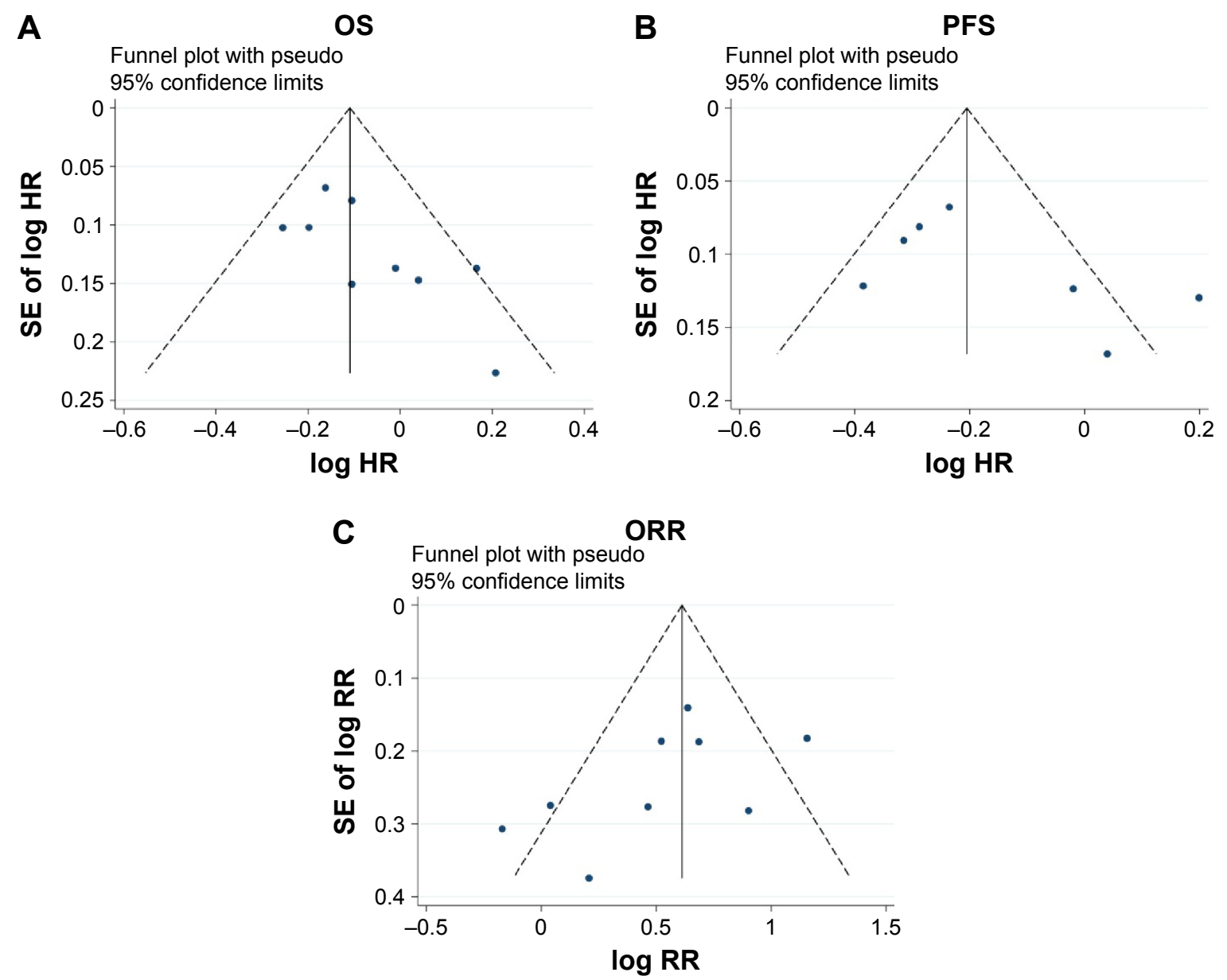

Figure 5 Funnel plot of publication bias in the meta-analysis.

Notes: (A) Overall survival (OS), (B) progression-free survival (PFS), and (C) overall response rate (ORR).

Abbreviations: HR, hazard ratio; SE, standard error; RR, risk ratio.

compared with single-agent therapy (Figure 2A and B) in the anthracyline- and taxane-pretreated breast cancer patients. The HR of 0.90 translates into a $10 \%$ reduction in the risk of death in patients receiving doublet therapy compared with those treated with single agent alone. Moreover, it should

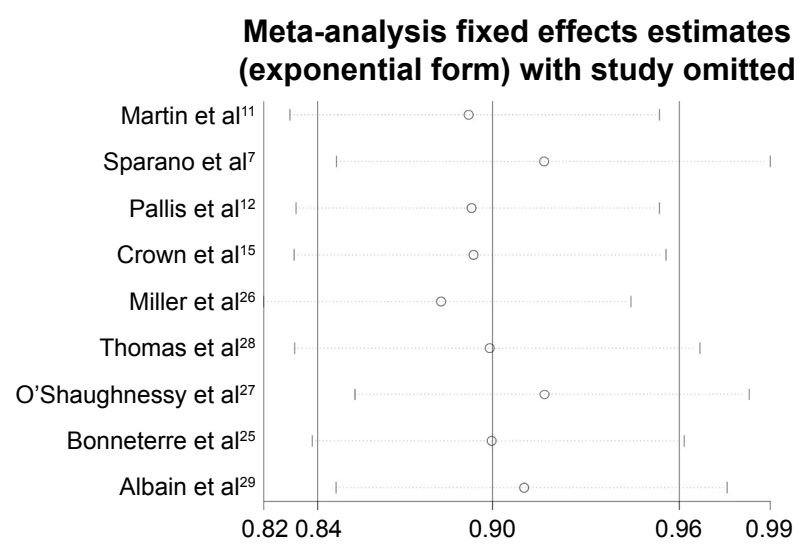

Figure 6 Sensitivity analysis of overall survival in this meta-analysis. be noted that all of our eligible trials did not specify the HER-2 status of the patients. And the survival information concerning the HER-2 status subgroups was not reported. Not to mention that the HER-2 data of two studies was unavailable..$^{25,27}$ Therefore, our meta-analysis is based on the general breast cancer population. Of course, clinical trials aimed at analysing the HER-2-negative or -positive subgroups are warranted in future.

On the basis of the promising results described previously, we demonstrated that combination therapy might represent a valuable option in the treatment of anthracycline- and taxanepretreated $\mathrm{MBC}$ patients. However, the toxicity and safety should be taken into account as well. For MBC patients with limited anticipated survival, impairment of the quality of life and increase in uncontrollable toxicity often outweigh the marginal efficacious benefit, leading to patient withdrawals or even to toxicity-associated deaths. ${ }^{32}$ In this meta-analysis, most of the grade 3-4 hematological toxicities were more frequently observed in the combination therapy group compared 
with single-agent group. In addition, a greater incidence of gastrointestinal toxicities such as nausea and stomatitis was also observed in the combination agents group. However, the incidences of toxicity-associated deaths between the two treatment groups were comparable (Table 2). This suggested that the majority of treatment-related toxicities were acceptable and manageable, although they were more common in the doublet agents group.

As mentioned earlier, OS, PFS, and ORR did not significantly improve with the targeted drug-based combination therapy (sunitinib or bevacizumab) compared with singleagent therapy (OS: HR 1.08, 95\% CI 0.89-1.31, $P=0.365$; PFS: HR 1.09, 95\% CI 0.88-1.35, $P=0.433$; Figure 2A and B). Therefore, targeted therapy plus chemotherapy should not be considered as the first treatment option in the anthracycline- and taxane-pretreated MBC patients. Moreover, ixabepilone-based combination therapy was also evaluated in our subgroup analysis. Consistent with a previous study, ${ }^{33}$ we also found that the ixabepilone-based doublets led to superior OS, superior PFS, and superior ORR compared with single-agent chemotherapy alone, with a manageable safety profile (Figures S1 and S2). These results taken together further supported the notion that doublet therapy can be considered for the heavily pretreated $\mathrm{MBC}$, while efficacies may depend on different drug combinations. For example, the targeted therapy plus chemotherapy must be carefully chose.

\section{Conclusion}

Indeed, we should acknowledge that, some limitations of this meta-analysis should be taken into consideration when interpreting the results. First, although strict inclusion criteria were set, the different study designs including treatment regimens, drug doses, and patient selections might still contribute to the observed heterogeneity. The cumulative dose of anthracyclines and taxanes was also not homogeneous. Moreover, the lines of the therapy and whether these agents were given together or in sequential administration in MBC were not always specified. Some patients received the treatments as the first-line salvage therapy, while some others received the treatments as the second- or the third-line therapy. We can not rule out the possibility that the sequential administration exerts similar advantages compared with single drug alone. Therefore, clinical investigations and analysis of these issues are warranted. Second, different drug combinations might partly result in bias of our findings. Especially for the targeted drug-based combination, only two RCTs ${ }^{15,26}$ (included only two drugs: sunitinib and bevacizumab) were available, and therefore, the conclusion was immature to some extent. Sensitivity analysis also indicated that studies with ixabepilone combination therapy obviously influenced the reliability of the overall estimates of our meta-analysis (Figure 6). Third, despite that adequate randomization was used, the results may have been overestimated because a blind investigational method was not applied. The present meta-analysis was performed based on the HRs and CIs reported directly or indirectly, whereas the information of individual patients was unavailable. Therefore, a meta-analysis based on more clearly individual treatment benefits was unable to be conducted. Fourth, the small sample size of the trials included in this meta-analysis inevitably imposed restrictions on the reliability of the data. In particular, a limited number of patients might have precluded some kinds of infrequent treatment-related toxicities. Finally, all of the studies in this meta-analysis were derived from those undertaken in the West. Thus, more studies based on Asian populations are urgently needed to confirm these results.

\section{Acknowledgments}

This work was supported by two grants from National Natural Science Foundation of China (nos 81172522 and 81301858).

\section{Disclosure}

The authors report no conflicts of interest in this work.

\section{References}

1. Siegel R, Ma J, Zou Z, Jemal A. Cancer statistics, 2014. CA Cancer J Clin. 2014;64(1):9-29.

2. Piccart-Gebhart MJ, Burzykowski T, Buyse M, et al. Taxanes alone or in combination with anthracyclines as first-line therapy of patients with metastatic breast cancer. J Clin Oncol. 2008;26(12):1980-1986.

3. Jerusalem G, Rorive A, Collignon J. Chemotherapy options for patients suffering from heavily pretreated metastatic breast cancer. Future Oncol. 2015;11(12):1775-1789.

4. Andreopoulou E, Sparano JA. Chemotherapy in patients with anthracycline- and taxane-pretreated metastatic breast cancer: an overview. Curr Breast Cancer Rep. 2013;5(1):42-50.

5. Toi M, Saeki T, Aogi K, et al. Late phase II clinical study of vinorelbine monotherapy in advanced or recurrent breast cancer previously treated with anthracyclines and taxanes. Jpn J Clin Oncol. 2005; 35(6):310-315

6. Seo HY, Lee HJ, Woo OH, et al. Phase II study of vinorelbine monotherapy in anthracycline and taxane pre-treated metastatic breast cancer. Invest New Drugs. 2011;29(2):360-365.

7. Sparano JA, Vrdoljak E, Rixe O, et al. Randomized phase III trial of ixabepilone plus capecitabine versus capecitabine in patients with metastatic breast cancer previously treated with an anthracycline and a taxane. J Clin Oncol. 2010;28(20):3256-3263.

8. O'Sullivan Coyne G, Walsh J, Kelly CM. Effectiveness and safety of eribulin mesylate: a new therapeutic option in the treatment of metastatic breast cancer. Expert Opin Drug Saf. 2012;11(4):643-650. 
9. Fumoleau P, Largillier R, Clippe C, et al. Multicentre, phase II study evaluating capecitabine monotherapy in patients with anthracycline- and taxane-pretreated metastatic breast cancer. Eur J Cancer. 2004;40(4): 536-542.

10. Abstracts of the 31 st ESMO (European Society for Medical Oncology) Congress, 29 September-3 October 2006, Istanbul, Turkey. Ann Oncol. 2006;17(suppl 9):ix7-ix326.

11. Martin M, Ruiz A, Munoz M, et al; Spanish Breast Cancer Research Group, (GEICAM) trial. Gemcitabine plus vinorelbine versus vinorelbine monotherapy in patients with metastatic breast cancer previously treated with anthracyclines and taxanes: final results of the phase III Spanish Breast Cancer Research Group (GEICAM) trial. Lancet Oncol. 2007; $8(3): 219-225$

12. Pallis AG, Boukovinas I, Ardavanis A, et al. A multicenter randomized phase III trial of vinorelbine/gemcitabine doublet versus capecitabine monotherapy in anthracycline- and taxane-pretreated women with metastatic breast cancer. Ann Oncol. 2012;23(5):1164-1169.

13. Lee KS, Park IH, Nam BH, Ro J. Phase II study of irinotecan plus capecitabine in anthracycline- and taxane- pretreated patients with metastatic breast cancer. Invest New Drugs. 2013;31(1):152-159.

14. Brito LG, de Andrade JM, Lins-Almeida T, et al. Safety and efficacy of gemcitabine plus cisplatin combination in pretreated metastatic breast cancer patients. Med Oncol. 2012;29(1):33-38.

15. Crown JP, Dieras V, Staroslawska E, et al. Phase III trial of sunitinib in combination with capecitabine versus capecitabine monotherapy for the treatment of patients with pretreated metastatic breast cancer. J Clin Oncol. 2013;31(23):2870-2878.

16. Moher D, Liberati A, Tetzlaff J, Altman DG. Preferred reporting items for systematic reviews and meta-analyses: the PRISMA statement. J Clin Epidemiol. 2009;62(10):1006-1012.

17. Jadad AR, Moore RA, Carroll D, et al. Assessing the quality of reports of randomized clinical trials: is blinding necessary? Control Clin Trials. 1996;17(1):1-12.

18. Parmar MK, Torri V, Stewart L. Extracting summary statistics to perform meta-analyses of the published literature for survival endpoints. Stat Med. 1998;17(24):2815-2834.

19. Tierney JF, Stewart LA, Ghersi D, Burdett S, Sydes MR. Practical methods for incorporating summary time-to-event data into metaanalysis. Trials. 2007;8:16.

20. Zintzaras E, Ioannidis JP. Heterogeneity testing in meta-analysis of genome searches. Genet Epidemiol. 2005;28(2):123-137.

21. DerSimonian R, Laird N. Meta-analysis in clinical trials. Control Clin Trials. 1986;7(3):177-188.
22. Mantel N, Haenszel W. Statistical aspects of the analysis of data from retrospective studies of disease. J Natl Cancer Inst. 1959;22(4):719-748.

23. Yusuf S, Peto R, Lewis J, Collins R, Sleight P. Beta blockade during and after myocardial infarction: an overview of the randomized trials. Prog Cardiovasc Dis. 1985;27(5):335-371.

24. Begg CB, Mazumdar M. Operating characteristics of a rank correlation test for publication bias. Biometrics. 1994;50(4):1088-1101.

25. Bonneterre J, Roché H, Monnier A, et al. Docetaxel vs 5-fluorouracil plus vinorelbine in metastatic breast cancer after anthracycline therapy failure. Br J Cancer. 2002;87(11):1210-1215.

26. Miller KD, Chap LI, Holmes FA, et al. Randomized phase III trial of capecitabine compared with bevacizumab plus capecitabine in patients with previously treated metastatic breast cancer. J Clin Oncol. 2005;23(4):792-799.

27. O'Shaughnessy J, Miles D, Vukelja S, et al. Superior survival with capecitabine plus docetaxel combination therapy in anthracyclinepretreated patients with advanced breast cancer: phase III trial results. J Clin Oncol. 2002;20(12):2812-2823.

28. Thomas ES, Gomez HL, Li RK, et al. Ixabepilone plus capecitabine for metastatic breast cancer progressing after anthracycline and taxane treatment. J Clin Oncol. 2007;25(33):5210-5217.

29. Albain KS, Nag SM, Calderillo-Ruiz G, et al. Gemcitabine plus Paclitaxel versus Paclitaxel monotherapy in patients with metastatic breast cancer and prior anthracycline treatment. J Clin Oncol. 2008;26(24): 3950-3957.

30. O'Shaughnessy J. Extending survival with chemotherapy in metastatic breast cancer. Oncologist. 2005;10(suppl 3):20-29.

31. Qi WX, Tang LN, He AN, Shen Z, Yao Y. Comparison between doublet agents versus single agent in metastatic breast cancer patients previously treated with an anthracycline and a taxane: a meta-analysis of four phase III trials. Breast. 2013;22(3):314-319.

32. Christopher T, Gradishar WJ, O'Shaughnessy JA, Bramsen B, Lurie RH. Clinical roundtable monograph: effective management of quality of life in metastatic breast cancer. Clin Adv Hematol Oncol. 2014; 12(2 suppl 4):1-14. quiz 15.

33. Roché H, Conte P, Perez EA, et al. Ixabepilone plus capecitabine in metastatic breast cancer patients with reduced performance status previously treated with anthracyclines and taxanes: a pooled analysis by performance status of efficacy and safety data from 2 phase III studies. Breast Cancer Res Treat. 2011;125(3):755-765. 


\section{Supplementary materials}

A

os

Study ID

$\mathrm{HR}(95 \% \mathrm{Cl})$

$\%$ weight

1

Thomas et $\mathrm{al}^{28}$

Sparano et $\mathrm{al}^{7}$

Subtotal $\left(I^{2}=0.0 \%, P=0.584\right)$

2

O'Shaughnessy et $\mathrm{al}^{27}$

Bonneterre et $\mathrm{al}^{25}$

Miller et $\mathrm{al}^{26}$

Martin et $\mathrm{al}^{11}$

Pallis et al $^{12}$

Crown et al ${ }^{15}$

Subtotal $\left(I^{2}=40.1 \%, P=0.138\right)$

Heterogeneity between groups: $P=0.238$

Overall $\left(I^{2}=30.3 \%, P=0.186\right)$

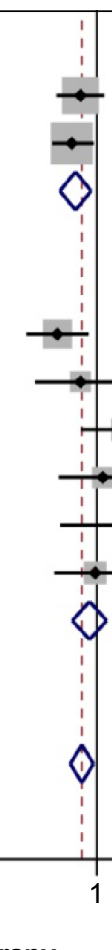

Favors

combination therapy
1
$\vdots$
1
1
1
1
1
1
1
1
0
1
1
1
1
1
1
1
0

PFS
$0.90(0.77,1.05) \quad 23.28$

$0.85(0.75,0.98) \quad 31.31$

$0.87(0.79,0.96) \quad 54.59$

$0.77(0.63,0.95) \quad 13.91$

$0.90(0.67,1.21) \quad 6.41$

$1.18(0.90,1.54) \quad 7.76$

$1.04(0.78,1.39) \quad 6.71$

$1.23(0.79,1.92) \quad 2.84$

$0.99(0.76,1.30) \quad 7.77$

$0.95(0.85,1.07) \quad 45.41$

$0.91(0.84,0.98) \quad 100$

B

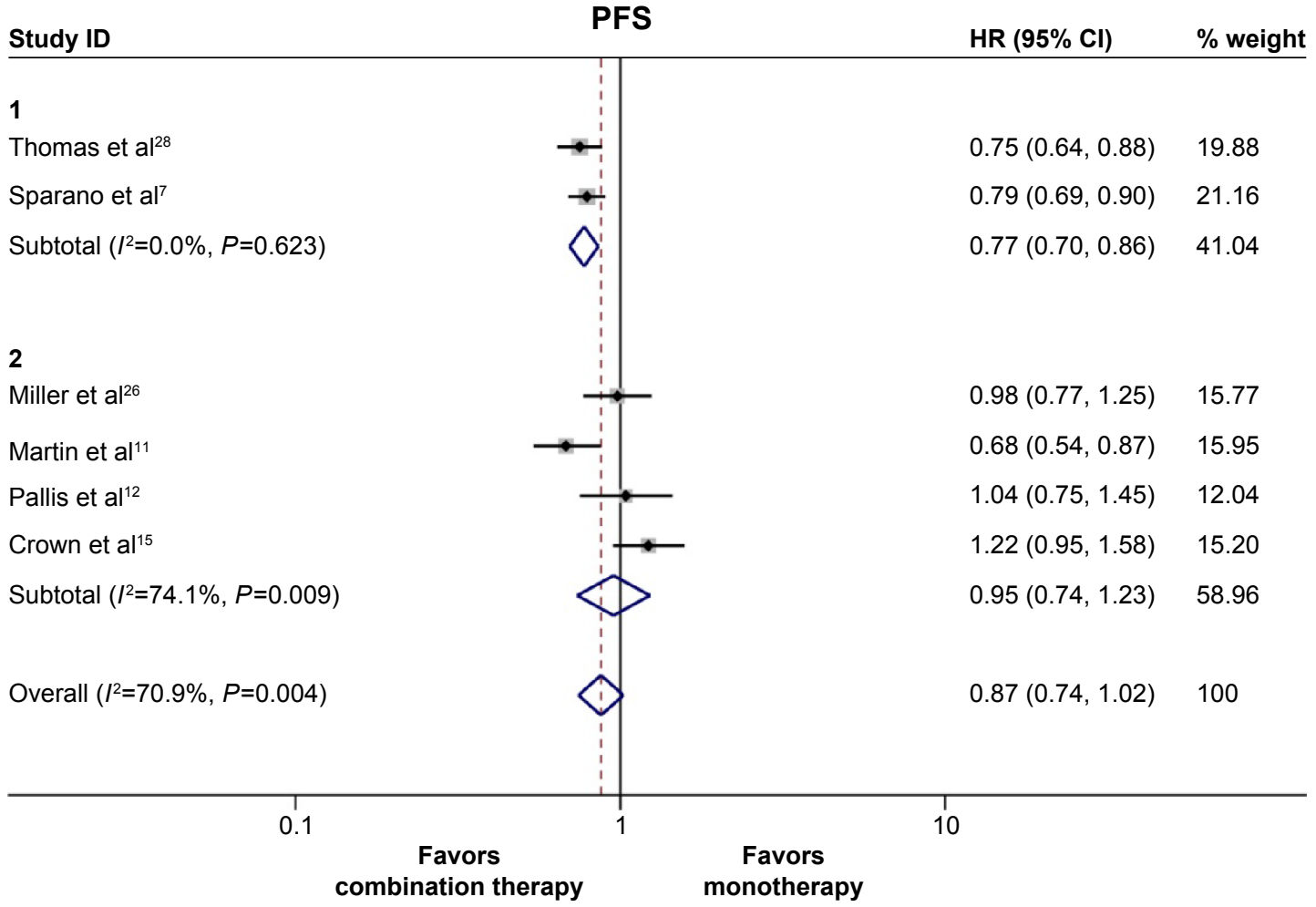

Figure SI The pooled hazard ratio (HR) and $95 \% \mathrm{Cl}$ for survival following ixabepilone-based doublet agents therapy and single-agent therapy.

Notes: The size of the square box is proportional to the weight that each study contributes in the meta-analysis. The overall estimate and confidence interval are marked by a diamond. Symbols on the right of the solid line indicate HR $>$ I, and symbols on the left of the solid line indicate HR $<$ I. (A) Overall survival. (B) Progression-free survival. For the subgroups, group I represents the studies that compared the ixabepilone-based doublets with single-agent therapy, and group 2 represents the studies that compared the other regimens with single-agent therapy. The HRs and $95 \% \mathrm{Cls}$ for OS and PFS were calculated with a fixed effect model. Weights are from random effect model. Abbreviations: $\mathrm{Cl}$, confidence interval; OS, overall survival; PFS, progression-free survival. 


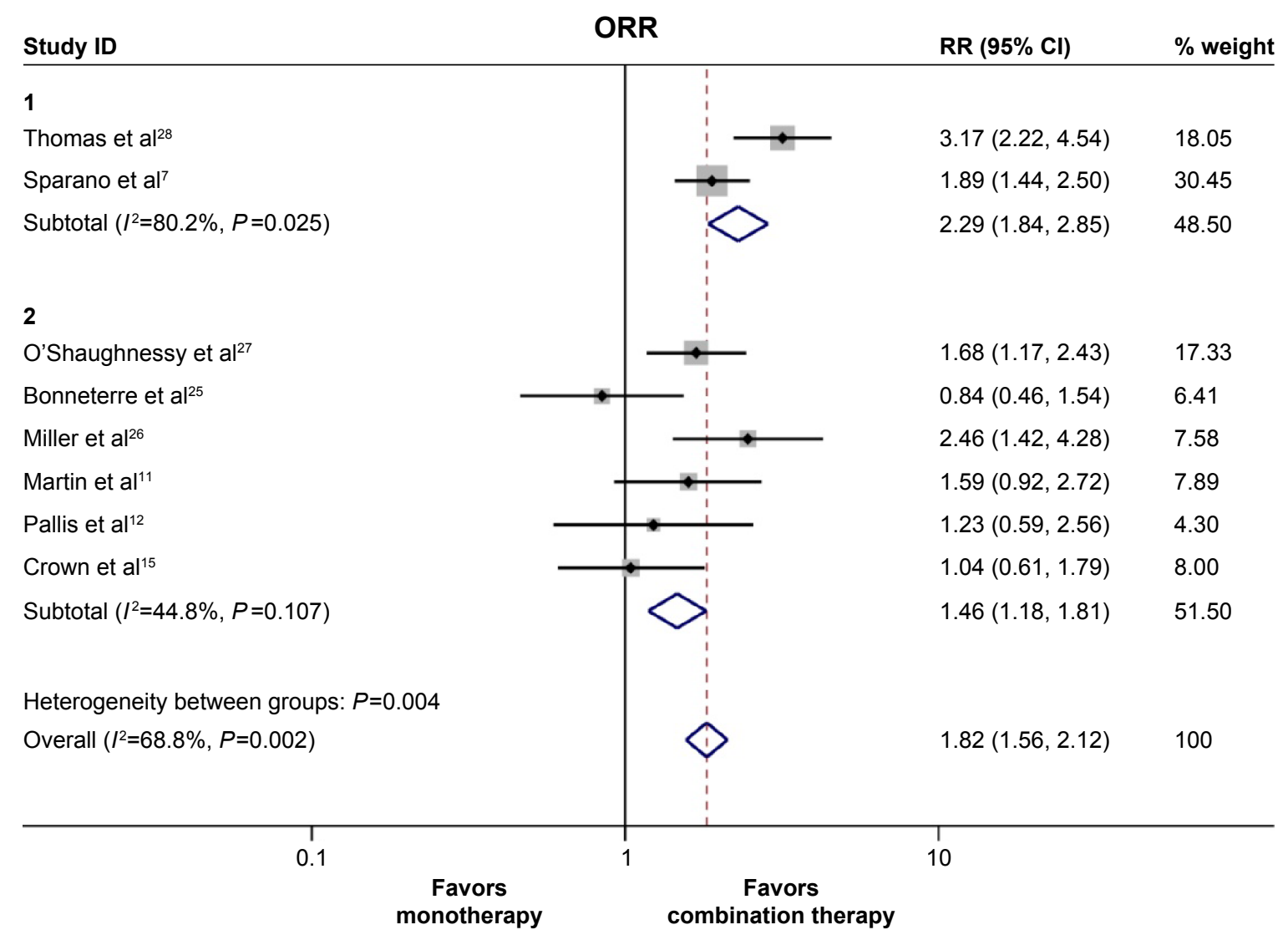

Figure S2 Pooled risk ratio (RR) and $95 \% \mathrm{Cl}$ for overall response rate of ixabepilone-based combination versus single-agent therapy.

Notes: The size of the square box is proportional to the weight that each study contributes in the meta-analysis. The overall estimate and confidence interval are marked by a diamond. Symbols on the right of the solid line indicate RR $>$ I, and symbols on the left of the solid line indicate RR $<$ I. The RR and $95 \% \mathrm{Cl}$ were calculated with a random effect model. For the subgroups, group I represents the studies that compared the ixabepilone-based doublets with single-agent therapy, and group 2 represents the studies that compared the other regimens with single-agent therapy. The RR and $95 \% \mathrm{Cl}$ were calculated with a fixed effect model.

Abbreviations: $\mathrm{Cl}$, confidence interval; ORR, overall response rate.

\section{Publish your work in this journal}

OncoTargets and Therapy is an international, peer-reviewed, open access journal focusing on the pathological basis of all cancers, potential targets for therapy and treatment protocols employed to improve the management of cancer patients. The journal also focuses on the impact of management programs and new therapeutic agents and protocols on patient perspectives such as quality of life, adherence and satisfaction. The manuscript management system is completely online and includes a very quick and fair peer-review system, which is all easy to use. Visit http://www.dovepress.com/testimonials.php to read real quotes from published authors. 